\title{
Postcards from the Middle West: A Photo Essay Philip Kobylarz
}

WHY THEY REFER TO IT as the (Mid)West is a mystery that long ago expired. With its small towns steepled with white-washed churches and downtowns made of locally quarried stone, this region resembles more closely the East coast. Only its ocean disappeared millions of years ago. Now the waveless sky, which is mostly a crucible of clouds, takes its place. It is a location that is neither here nor there.

A land whose timelessness is altogether rooted in the eternity of the cycle. The changing seasons. Smell of leaves burning in October. December's wind chill freezing your spit into glass. The floating sex of pollen and cut grass in the spring. The Reaper's solemn duty of the Harvest.

A place of slow-running rivers; the half-made gesture of hills that barely promise distance and can only mimic the stance of mountains. Limestone cliffs break apart on either side of the Mississippi: the guts of the tabletop checkerboard of farmland that stretches out for what might as well be forever. There are caves that lead to nowhere. Deer and fox use the cities at night as wilderness crossings.

Metropolises evacuated after five o'clock reign over the prairie as monuments to stillness. Chicago is at ease as the rival of New York. St. Louis at the same odds with Philadelphia. It's almost as if the people don't want anything here to get too big. Or out of hand. The countryside carved into geometric gridlock attests to some fact of matter.

A gothic Victorian house surrounded by spruce and pine occupies a hilltop. A field of cows. The choking ether of near ninety percent humidity. Weeds spawn weeds. A stave silo in a fallow field buckles to its knees. A red-tailed hawk's cry pierces the silence while it circles within a thermal overhead. A crude two by four-ed sign off the highway reads Time Ends Eternity Where. It makes sense in the rearview. Just so many words. 


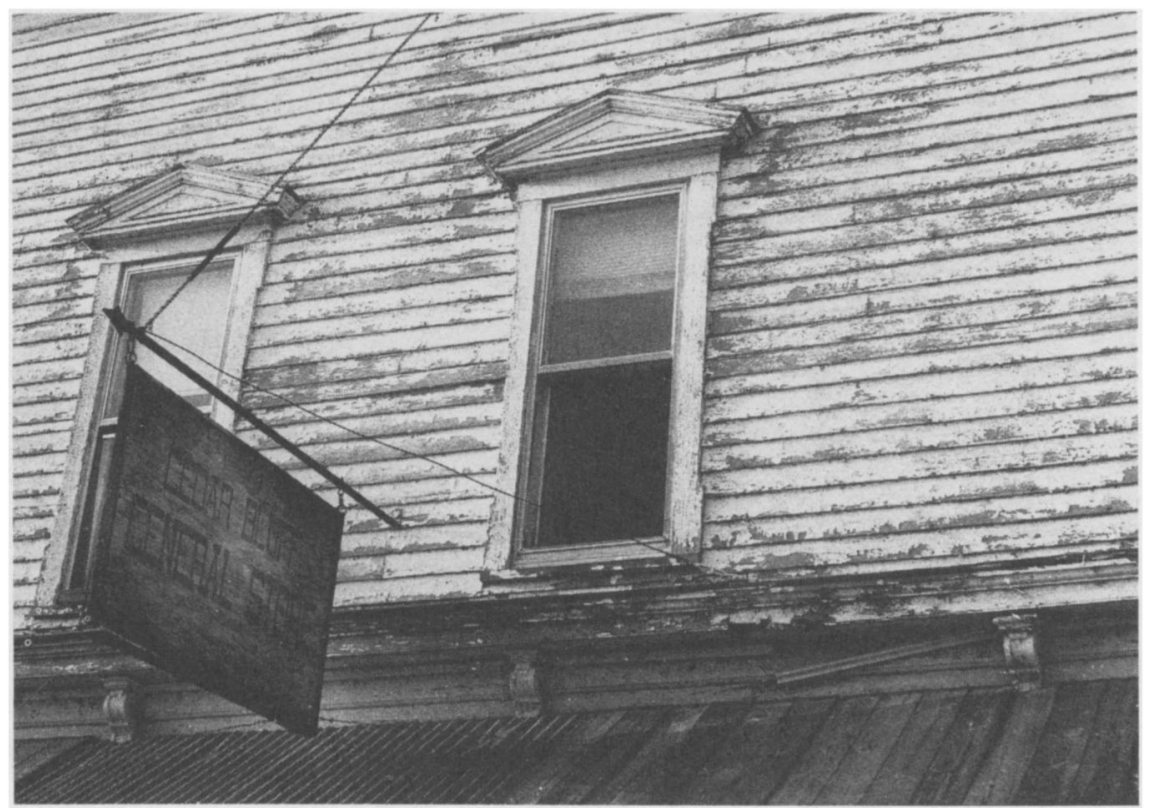

General Store (for rent)

Cedar Bluff, IA

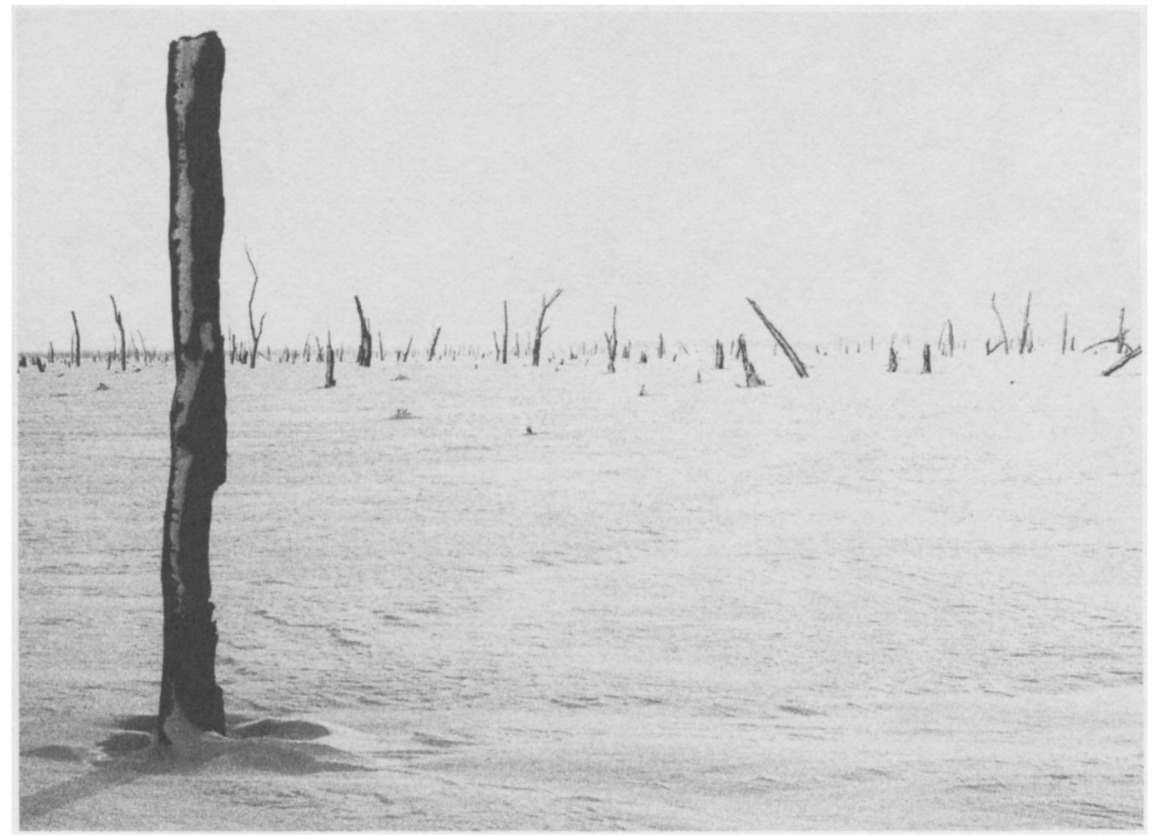

In the Garden of the Monoliths 


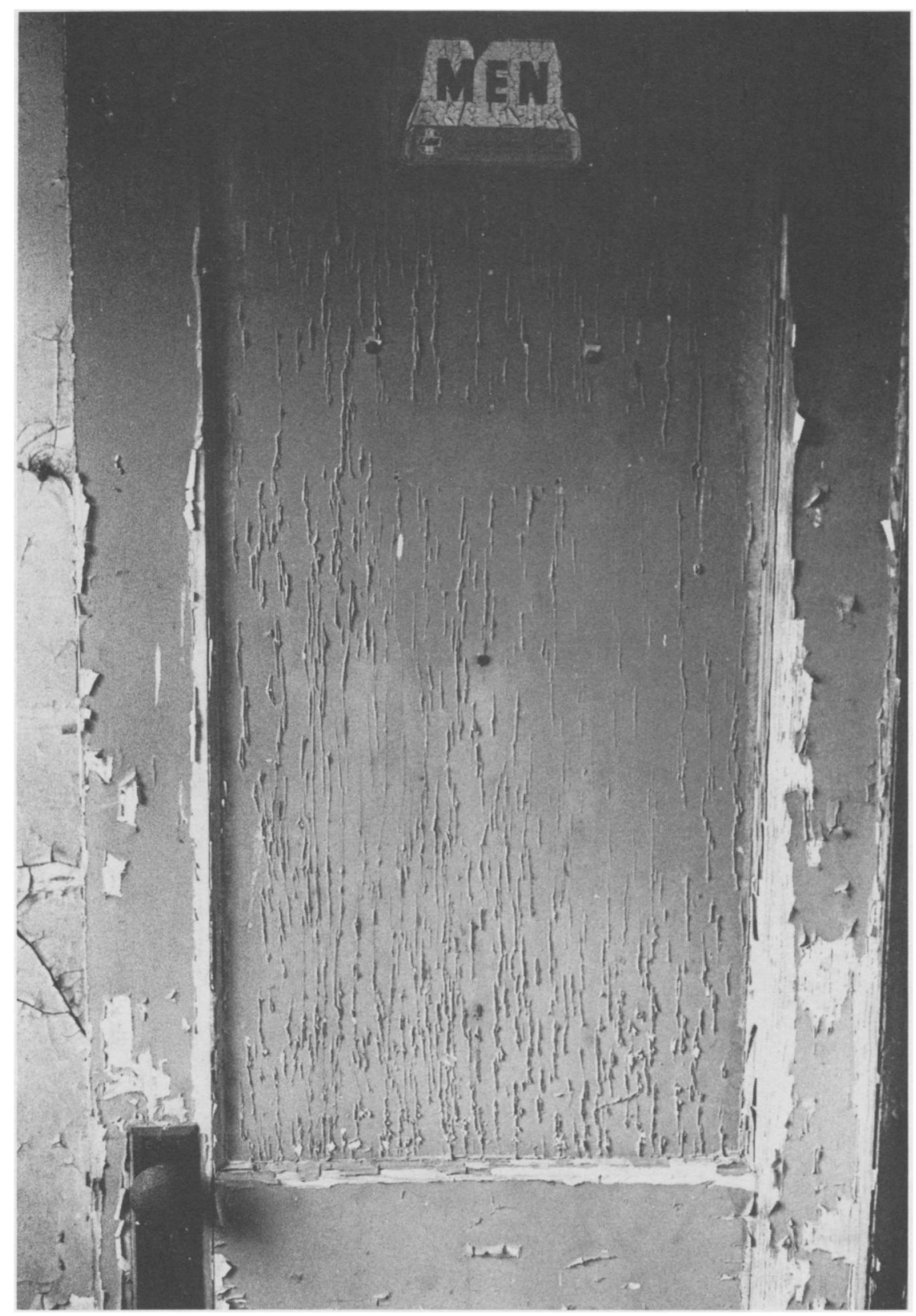

\section{Abandoned Door}




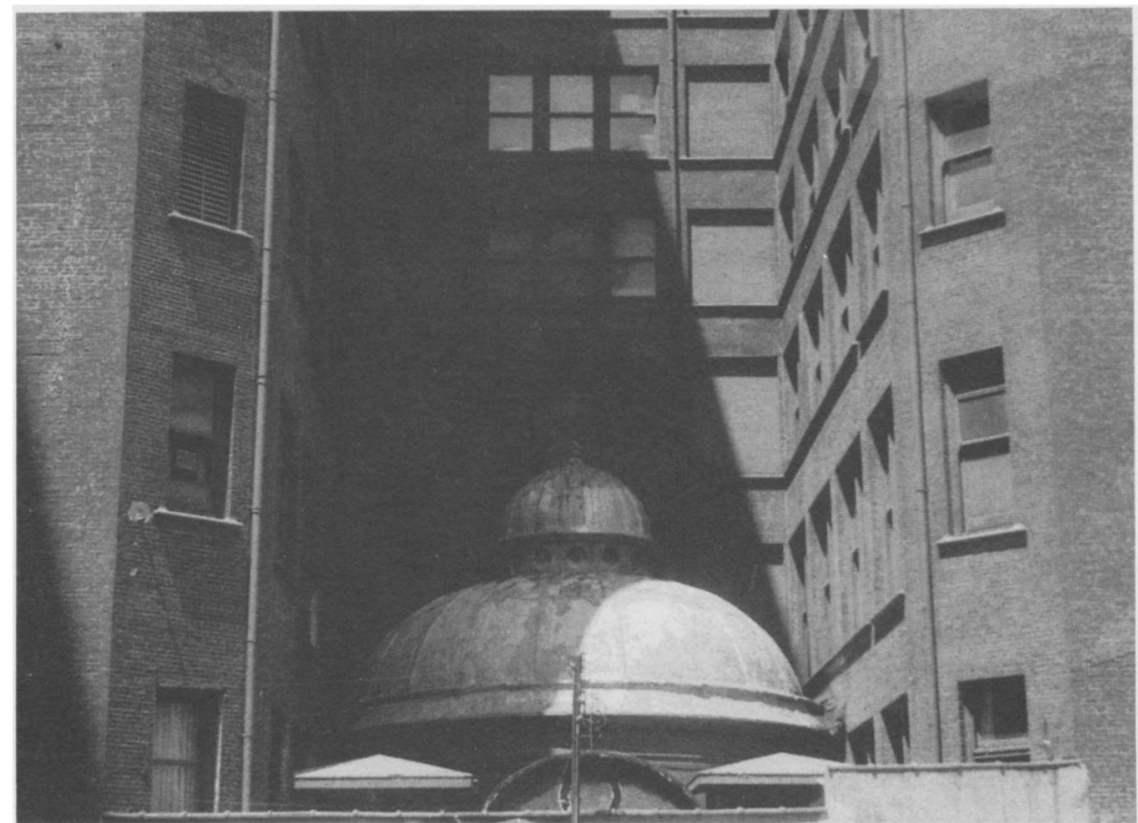

St. Louis mosque

Olive Street, 1991

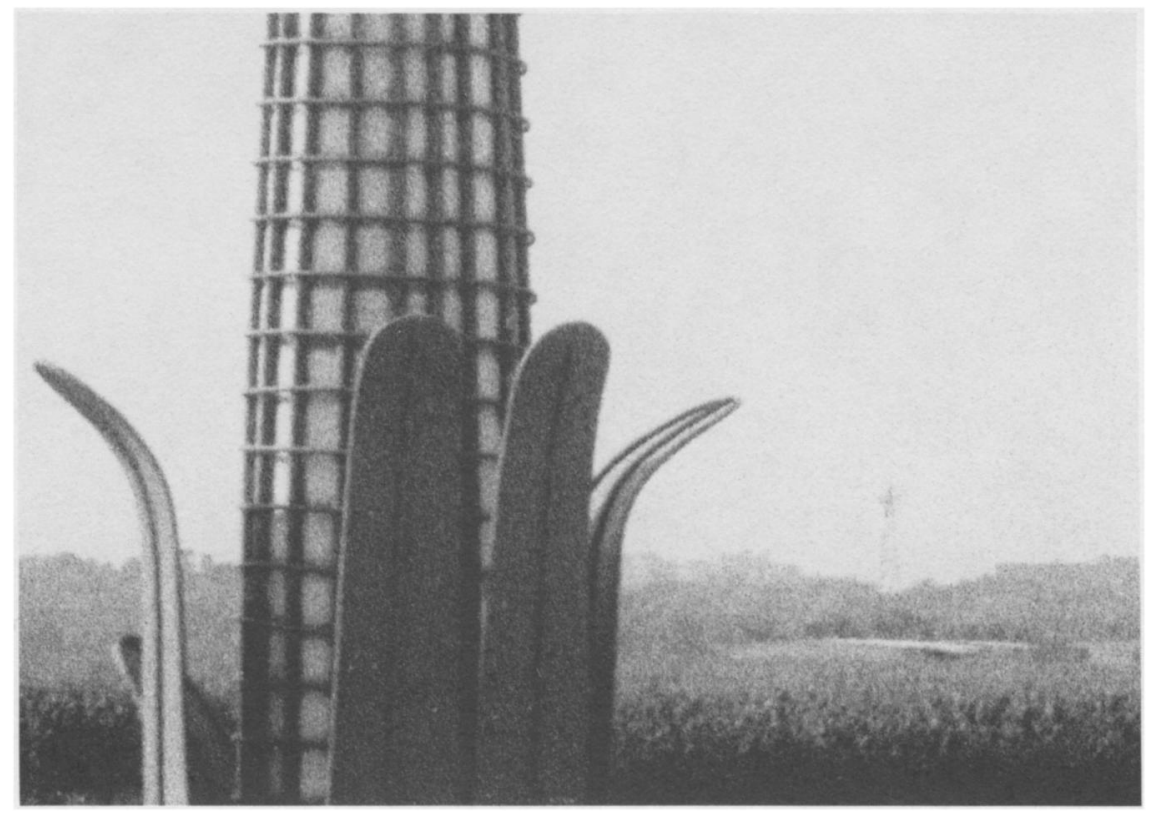

Among the Alien Corn 


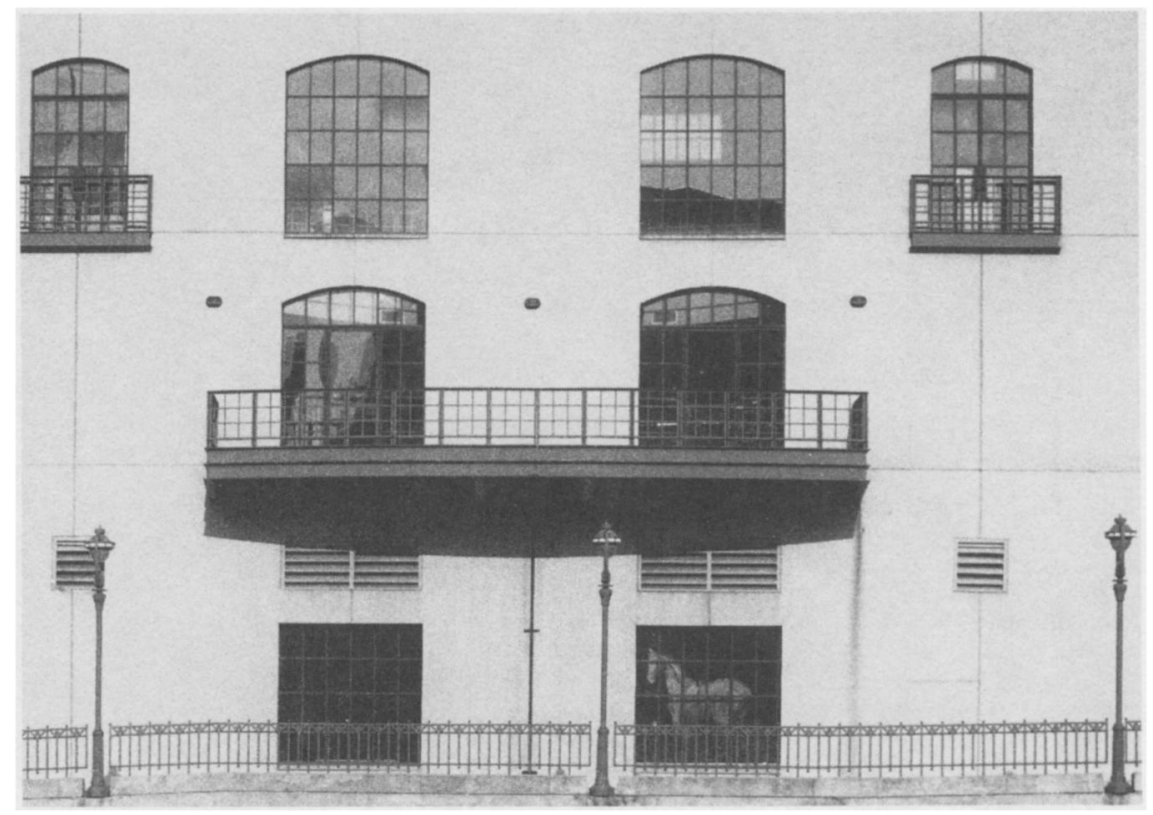

\section{The Carriage House}

Milwaukee

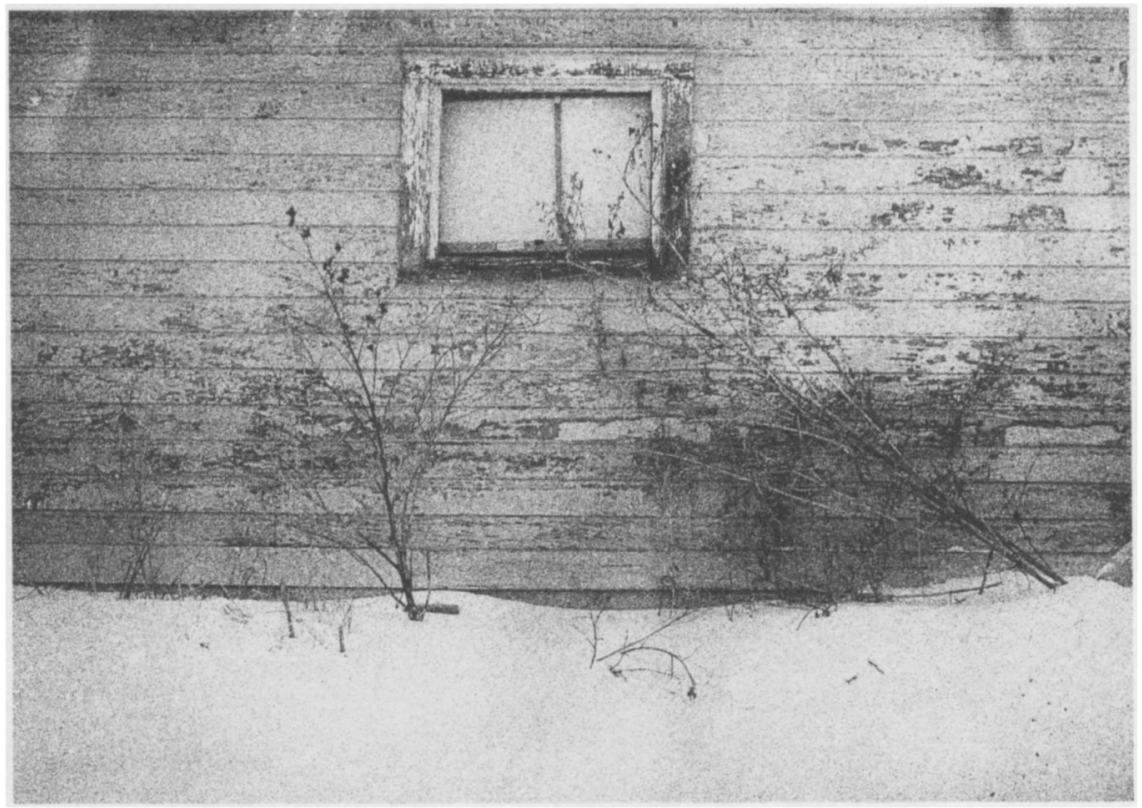

\section{November Canvass}




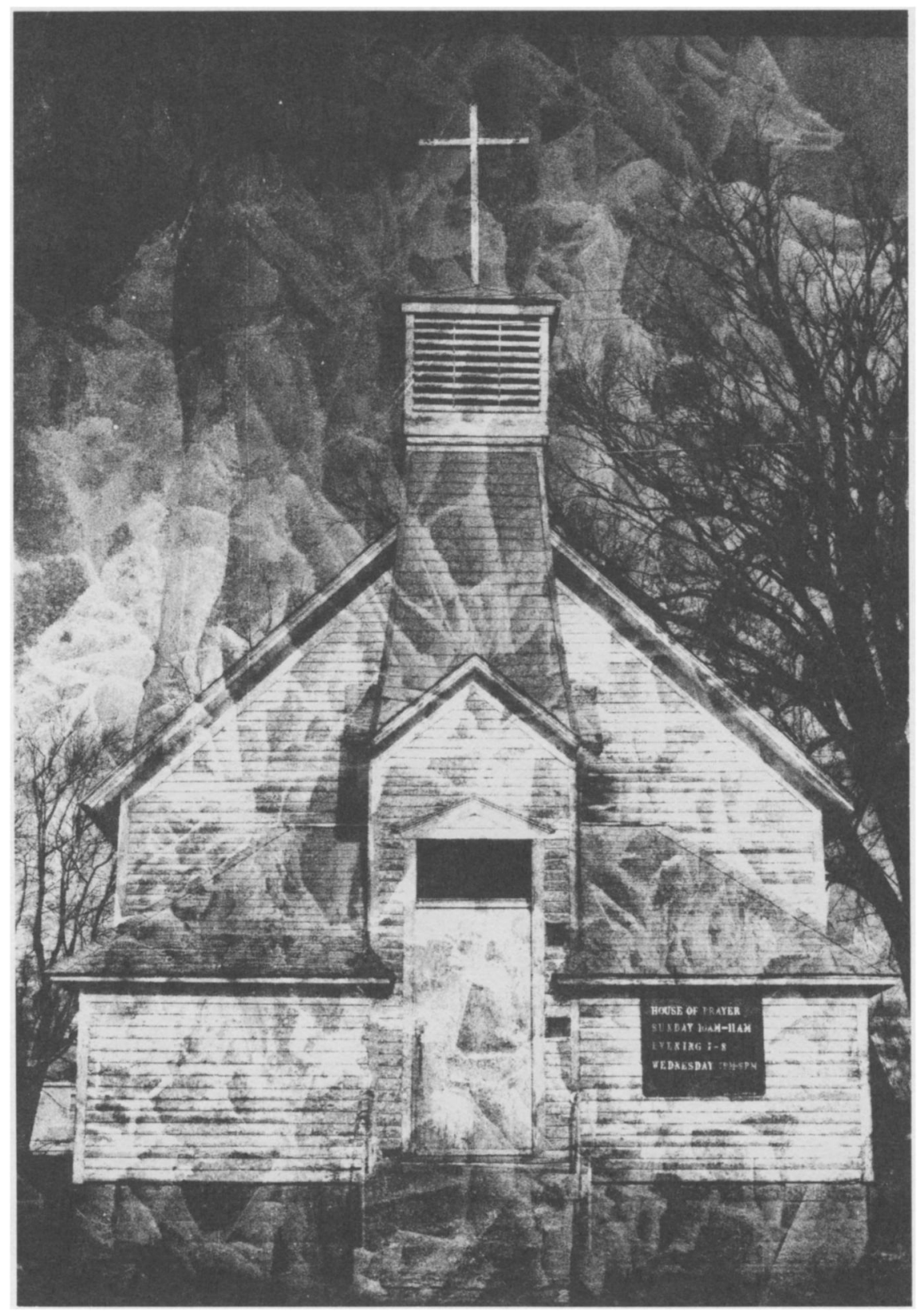

Self-titled

Montpelier, IA 